\title{
Antibiotic prophylaxis in acute cholecystectomy revisited: results of a double-blind randomised controlled trial
}

\author{
Gona Jaafar $^{1}$ (D) $\cdot$ Gabriel Sandblom $^{2}$ (D) Lars Lundell $^{3,4} \cdot$ Folke Hammarqvist $^{1}$ (D)
}

Received: 8 July 2020 / Accepted: 23 August 2020 / Published online: 29 August 2020

(C) The Author(s) 2020

\begin{abstract}
Purpose Evidence supporting the value of preoperative antibiotic prophylaxis (PAP) in surgery for acute cholecystitis is lacking. This study aimed to shed light on whether PAP in acute cholecystectomy for cholecystitis reduces the postoperative infectious complication (PIC) rate. Secondary outcomes were the prevalence of bacteriobilia, CRP values and leucocyte counts.

Methods The study was performed as a single-centre, double-blinded, placebo-controlled, randomised study. Patients with acute cholecystitis amenable for acute laparoscopic cholecystectomy were randomly assigned to either PAP (piperacillin/tazobactam) or placebo, and the subsequent clinical course was followed.

Results A total of 106 patients were enrolled, 16 of whom were excluded due to protocol violation. PIC developed in 22 of the 90 patients included with no significant difference between the PAP and placebo groups ( 8 patients in the PAP group and 14 in the placebo arm, $p=0.193$ ). The PIC rate was significantly higher in patients with a raised CRP at randomisation and on the day of surgery and in cases of conversion to an open procedure $(p=0.008,0.004$ and 0.017 , respectively) but with no differences between the study groups.

Conclusion PAP does not affect the risk for PIC in patients with acute cholecystitis. The major risk factors determining PIC in these patients need defining, in particular, the impact of bacteriobilia.

Trial registration The study was registered at clinicaltrials.gov (NCT02619149) December 2, 2015.
\end{abstract}

Keywords Acute cholecystitis - Laparoscopic cholecystectomy · Antibiotic prophylaxis · Postoperative complication · Postoperative infectious complication · Bacteriobilia

\section{Introduction}

The standard treatment for mild to moderate acute cholecystitis is early laparoscopic cholecystectomy [1-4]. Given that the overall complication rate after cholecystectomy is approximately $11 \%$ (Swedish GallRiks register) [5], the risk for

Gabriel Sandblom

gabriel.sandblom@ki.se

$1 \quad$ Subject Trauma Reparative Medicine, CLINTEC, Karolinska University Hospital, 14186 Stockholm, Sweden

2 Department of Clinical Science and Education, Department of Surgery, Södersjukhuset, Karolinska Institute, Stockholm, Sweden

3 Department of Surgery, CLINTEC, Karolinska Institute, Stockholm, Sweden

4 Department of Surgery, Odense University Hospital, J.B. Winsloews Vej 4, 5000 Odense, Denmark postoperative infectious complications (PIC) after gallstone surgery is small but not negligible. The risk appears to be enhanced by the severity of the ongoing cholecystitis, and PIC has been shown to occur in $17 \%$ or more of patients with grades I and II acute cholecystitis [6]. The pathogenesis of PIC in connection with elective and acute operations for gallstone disease includes contamination with infected bile. Under normal conditions, bile in the gallbladder and the biliary tree is sterile [7]. Obstruction of the outflow of bile by gallstone(s) initiates an inflammatory process that eventually leads to bacterial colonisation. In acute cholecystitis, the bile becomes colonised in 35-60\% of the cases [8], the most common agents being gram-negative organisms (Escherichia coli 3144\%, Klebsiella 9-20\%) and Enterococci (3-34\%) [9, 10]. The mechanisms behind PIC are complex, and the details of the dynamics of colonisation are lacking. A recent retrospective study in Sweden showed that the only significant risk factor for PIC (approximately 6\%) was a positive bile culture. Gender, age, ongoing or previous cholecystitis and 
preoperative antibiotic prophylaxis (PAP) had no impact on PIC [11]. However, despite the fact that surgery for acute cholecystitis is one of the most frequently performed procedures by general surgeons throughout the world, the use of PAP varies between hospitals and between surgeons, as recently shown in a nationwide study from Sweden [12]. Current guidelines do not support the routine use of PAP for elective cholecystectomy for uncomplicated gallstone disease [13-15], but surprisingly adherence to international guidelines is generally low in Europe [16, 17]. Lack of evidence-based guidelines could be a significant factor behind the disparities seen worldwide. Liang et al., in a systemic review and metaanalysis of low-risk patients undergoing elective laparoscopic cholecystectomy, concluded that PAP is safe and effective in reducing surgical site infections, global infections and postoperative length of hospital stay [18]. On the other hand, the 2013 Tokyo Guidelines (TG13) recommended PAP for interventions in acute cholangitis and cholecystitis only. Implementation of TG13 has led to uniform antibiotic treatment without increasing the risk for PIC [19]. However, the effectiveness of PAP in acute cholecystitis needs to be confirmed.

The aim of this study was to determine the effect of PAP with piperacillin/ tazobactam on PIC following acute cholecystectomy for mild to moderate cholecystitis (grades I and II according to TG18).

\section{Material and methods}

The study was undertaken as a prospective randomised double-blinded parallel group study at Karolinska University Hospital in Huddinge, with treatment allocation 1:1 between PAP and placebo.

\section{Eligibility criteria}

Inclusion criteria were clinical and radiological signs of acute cholecystitis grades I and II suitable for acute laparoscopic cholecystectomy and age $\geq 18$ years.

Exclusion criteria were ongoing septicaemia, pregnancy, bile duct obstruction, contraindication to laparoscopic surgery, treatment with antibiotic drugs within $24 \mathrm{~h}$ and symptom duration longer than 5 days.

Written and verbal informed consent was obtained after the decision to perform acute surgery had been taken. Inclusion and randomisation using sealed envelopes were usually performed the day before surgery, and the result was kept in a sealed envelope system by the research nurse. Blinding was performed in accordance with the recommendations of Probst et al. [20]. The intravenous drip set was covered by an opaque bag to maintain blinding, and a research nurse administered the infusion. The placebo infusion contained saline in a bottle indistinguishable from that containing the active drug. In exceptional situations, blinding was interrupted intraoperatively if the need to administer an antibiotic was considered imperative.

\section{Interventions and allocation}

The patients were randomised to either PAP (4 g piperacillin/ tazobactam) or placebo given as infusions before the procedure (79\% of PAP received one dose only prior to surgery). A research nurse administrated the study infusion according to allocation. The study infusion was started immediately after inclusion and continued until the procedure was completed. As the time between inclusion and the procedure varied, infusions were given over periods varying from less than an hour to $72 \mathrm{~h}$. The surgeon, ward staff, patients and researchers involved were all blinded to the allocation. Blood samples for CRP and leukocyte count were taken prior to and 2 days after the procedure by the research nurse.

\section{Bile sampling procedure}

During the laparoscopic procedure and under sterile conditions, bile was aspirated from the fundus of the gallbladder before the start of dissection and later from the cystic duct prior to cholangiography. The bile samples were transferred to both aerobic and anaerobic sealed bottles and sent to the hospital microbiology laboratory for culture. Patients with severely inflamed cholecystitis or need for intraoperative ERCP remained in the study for intention-to-treat analyses. At Karolinska University Hospital, the common routine following laparoscopic cholecystectomy is to retrieve the specimen using a bag.

\section{Outcomes}

The primary outcome was rate of PIC requiring antibiotic treatment or surgical intervention within 30 days postoperatively. PIC was defined as intra-abdominal abscess, sepsis, cholangitis, surgical site infection, pneumonia or urinary tract infection. Cases where antibiotic treatment was started without a clearly identified focus of infection were also classed as a PIC.

Secondary outcomes were prevalence of bacteriobilia, CRP values and leucocyte counts.

A research nurse invited all patients to a follow-up 30 days after cholecystectomy. Those who were unable to attend the follow-up were interviewed by telephone. If an event described at the telephone interview raised suspicion of a PIC, the patient's primary care records was reviewed. The 30-day PIC rate was determined from patient's records as well as from the patients' own reports. Data collection was performed 
by the research nurses and checked by the principal investigator for correction of missing data.

\section{Statistical analyses and ethics}

Chi-square and $t$ test were used to determine differences between the two groups regarding gender, operation approach, previous gallstone symptoms, postoperative complications and comorbidity. Mann-Whitney $U$ test was used for analysing non-parametric data (age, BMI, duration of symptoms, CRP, leucocyte count and body temperature). Bile culture data and inflammation markers were also analysed in relation to the occurrence of PIC. Separate per-protocol (PP) analyses were performed, i.e. excluding those who received perioperative antibiotic treatment despite placebo allocation, and those who were lost to follow-up.

\section{Sample size estimation:}

The sample size estimation was based on a hypothesis of superiority. Assuming that PAP would reduce the risk for PIC from 25 to $10 \%$, at least 77 patients were required to reach an $80 \%$ probability of detecting a statistically significant difference at the $p<0.05$ level (one-sided test). This hypothesised difference was considered to reflect a clinical effect that would be clinically relevant, although a lesser reduction in the incidence of PIC may have been relevant if more serious complications were to be considered.

\section{Results}

Altogether 106 patients were invited to participate in the study. Of these, 16 were excluded because of protocol violation (3 did not fulfil eligibility criteria, 1 withdrew consent, 8 due to postponed operation because of OR overload and 4 because the allocation envelope was missing). The remaining 90 patients were allocated to PAP or placebo (Fig. 1). The first patient was included on December 14, 2009, and the last to be included was followed until April 4, 2017. There were no differences between the groups regarding demographic and disease-specific characteristics (Table 1). Fifteen patients were given antibiotic treatment in the immediate postoperative period due to severe contamination of the abdomen, regardless of allocation. These were classified as PIC even though the decision to give antibiotic treatment was taken perioperatively whether prophylaxis had been given or not. Seventeen patients were lost to follow-up (Fig. 1). Four patients were

Table 1 Baseline characteristics

\begin{tabular}{|c|c|c|c|c|c|c|}
\hline \multirow[t]{2}{*}{ Variables } & \multicolumn{3}{|c|}{ Intention to treat analysis $(n 90)$} & \multicolumn{3}{|c|}{ Per protocol analysis ( $n$ 58) } \\
\hline & $\begin{array}{l}\text { Antibiotic }(\%) \\
42(47)\end{array}$ & $\begin{array}{l}\text { Placebo }(\%) \\
48(53)\end{array}$ & $p$ value & $\begin{array}{l}\text { Antibiotic }(\%) \\
29(50)\end{array}$ & $\begin{array}{l}\text { Placebo }(\%) \\
29(50)\end{array}$ & $p$ value \\
\hline Men $(\%)$ & $18(43)$ & $23(48)$ & 0.675 & $13(45)$ & $11(38)$ & 0.395 \\
\hline Age, years ( median, interquartile range) & $48.5(24)$ & $49(25)$ & 0.768 & $55(20)$ & $45(20)$ & 0.194 \\
\hline Body mass index ( median, interquartile range) & $27(7)$ & $28(6)$ & 0.874 & $28(9)$ & $27(5)$ & 0.428 \\
\hline Previous gallstone symptom (\%) & $13(31)$ & $11(30)$ & 0.476 & $10(34)$ & $6(21)$ & 0.379 \\
\hline No comorbidity (\%) & $13(31)$ & $21(44)$ & 0.277 & $8(28)$ & $14(48)$ & 0.175 \\
\hline Symptom duration (median, interquartile range) & $4(3)$ & $4(2)$ & 0.653 & $4(3)$ & $4(1)$ & 0.178 \\
\hline Method of approach $(\%)$ & & & 0.487 & & & 0.838 \\
\hline Laparoscopic & $37(88)$ & $38(79)$ & & $26(90)$ & $25(86)$ & \\
\hline Open & $1(2)$ & $3(6)$ & & $1(3)$ & $2(7)$ & \\
\hline Converted & $4(10)$ & $7(15)$ & & $2(7)$ & $2(7)$ & \\
\hline Body temperature inclusion day (median, interquartile range) & $37(21)$ & $37(1)$ & 0.810 & $37(2)$ & $37(2)$ & 0.433 \\
\hline C-reactive protein inclusion day (median, interquartile range) & $57(121)$ & $81(129)$ & 0.140 & $46(129)$ & $76(79)$ & 0.409 \\
\hline $\begin{array}{l}\text { White blood cell count inclusion day, } \times 10^{9} / 1 \text { (median, } \\
\text { interquartile range) }\end{array}$ & $10(5)$ & $12(7)$ & 0.105 & $9(6)$ & $10.5(8)$ & 0.600 \\
\hline Temp day 2 (median, interquartile range) & $37(2)$ & $37(0.5)$ & 0.398 & $37(2)$ & $37(1)$ & 0.893 \\
\hline C-reactive protein day 2 (median, interquartile range) & $760(175)$ & $80(118)$ & 0.650 & $56(151)$ & $70(52)$ & 0.844 \\
\hline $\begin{array}{l}\text { White blood cell count inclusion day, } \times 10^{9} / 1 \text { day } 2 \\
\text { (median, interquartile Range) }\end{array}$ & $10(7)$ & $11(5)$ & 0.536 & $8(8)$ & $10(4)$ & 0.545 \\
\hline Antibiotic treatment start postop (\%) & $5(12)$ & $10(21)$ & 0.396 & -- & -- & \\
\hline Postop complication (\%) & $8(19)$ & $14(29)$ & 0.193 & $3(10)$ & $3(10)$ & 0.665 \\
\hline
\end{tabular}


included based on primary intent to perform laparoscopic cholecystectomy, but the surgeon responsible for the procedure decided to do an open procedure for technical reasons. These patients remained in the study.

The rate of PIC was 24\% (9\% in the PAP arm and $16 \%$ in the placebo arm in the intention-to-treat and $5 \%$ in each group in the PP analysis). There was no significant difference in PIC between the groups regardless of the analytical approach ( $p=$ 0.193, Table 2).

Bile cultures were obtained in 48/90 (53\%) cases (evenly distributed between the study groups), and cultures were positive in 18/48 (38\%) (13 in the antibiotic group and 5 in the placebo group, $p=0.076)$. Gram-negative aerobes predominated in the cultures $(N=11)$, followed by gram-positive aerobes $(N=10)$, anaerobes $(N=3)$ and fungus $(N=1)$. PIC was numerically more common in those with a positive culture, but this did not reach statistical significance ( $p=0.054$, Table 3 ). There was no statistically significant association between the period of PAP and a positive bile culture.

CRP levels were significantly higher in patients with PIC, on both the day of randomisation $(p=0.008)$ and the day of surgery $(p=0.004)$. PIC was also more frequently seen in cases converted to an open procedure $(p=0.017)$, whereas patient comorbidity was not associated with PIC ( $p=0.075$, Table 2).

\section{Discussion}

In the present study, we were unable to detect any benefit of administrating PAP to reduce the risk for PIC after emergency cholecystectomy for grades I and II acute cholecystitis. Although we did not reach sufficient statistical power to detect a minor reduction in PIC rate, the absence of any significant impact speaks against the routine use of PAP. We observed a PIC rate of less than 20\%: $9 \%$ in the PAP arm and 16\% in the placebo arm in the ITT analysis and 5\% in each group in the PP analysis. These figures compare well with the expected PIC incidences seen after emergency cholecystectomy for cholecystitis [5]. It can be argued that the preconditions applied in the sample size calculation lack clinical relevance. On the other hand, there are no definited criteria for defining clinically important differences when assessing the effectiveness of PAP. The widespread overuse of antibiotics and

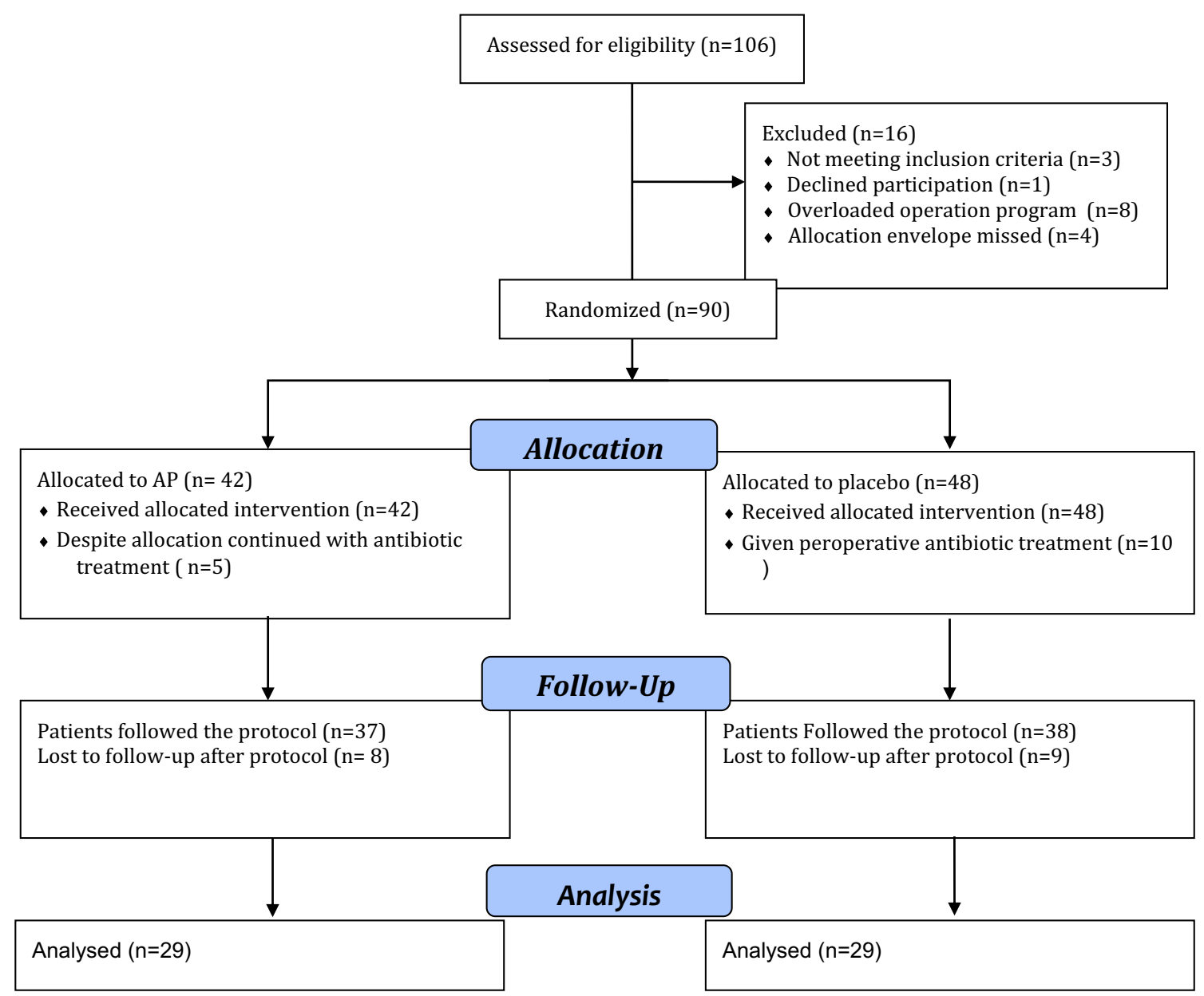

Fig. 1 Flow chart 
Table 2 Postoperative infectious complication

\begin{tabular}{|c|c|c|c|c|c|c|}
\hline \multirow[t]{2}{*}{ Variables } & \multicolumn{3}{|c|}{ Intention to treat analysis $(N=90)$} & \multicolumn{3}{|c|}{ Per protocol analysis $(N=58)$} \\
\hline & $\begin{array}{l}\text { Non-event (\%) } 68 \\
(76)\end{array}$ & Event (\%) $22(24)$ & $p$ value & $\begin{array}{l}\text { Non-event (\%) } 52 \\
(90)\end{array}$ & Event $(\%) 6(10)$ & $p$ value \\
\hline $\operatorname{Men}(\%)$ & $32(47)$ & $9(41)$ & 0.633 & $22(42)$ & $2(33)$ & 1.000 \\
\hline Age (interquartile range) & $47,5(24)$ & $58(25)$ & 0.081 & $49(22)$ & $59(26)$ & 0.301 \\
\hline BMI (interquartile range) & $27.4(6.5)$ & $27.7(6.3)$ & 0.936 & $28(7)$ & $28(6)$ & 0.861 \\
\hline Symptom duration (interquartile range) & $4(2)$ & $4(3)$ & 0.400 & $4(2)$ & $5(4)$ & 0.388 \\
\hline No comorbidity (\%) & $29(43)$ & $5(23)$ & $0.075(1 \mathrm{~s})$ & $21(40)$ & $1(17)$ & $0.253(1 \mathrm{~s})$ \\
\hline Operation method (\%) & & & $0.017 *$ & & & 0.335 \\
\hline Laparoscopic & $61(90)$ & $14(64)$ & & $46(88)$ & $5(83)$ & \\
\hline Open & $2(3)$ & $2(9)$ & & $2(4)$ & $1(17)$ & \\
\hline Converted & $5(7)$ & $6(27)$ & & $4(8)$ & $0(0)$ & \\
\hline $\begin{array}{l}\text { Body temperature allocation day } \\
\text { (median, interquartile range) }\end{array}$ & $37(2)$ & $37(1)$ & 0.513 & $37(1)$ & $37(1)$ & 0.409 \\
\hline $\begin{array}{l}\text { C-reactive protein allocation day } \\
\text { (median, interquartile range) }\end{array}$ & $57(121)$ & 124 (118) & $0.008^{*}$ & $57(94)$ & $131(123)$ & 0.102 \\
\hline $\begin{array}{l}\text { White blood cell count inclusion day, } \\
\times 10^{9} / 1 \text { (median, interquartile range) }\end{array}$ & $10(7)$ & $12(5)$ & 0.258 & $9(8)$ & $11(3)$ & 0.564 \\
\hline $\begin{array}{l}\text { Body temperature day } 1 \text { (median, } \\
\text { interquartile range) }\end{array}$ & $37(2)$ & $37(1)$ & 0.560 & $37(2)$ & $36(2)$ & 0.278 \\
\hline $\begin{array}{l}\text { C-reactive protein day } 1 \text { (median, } \\
\text { interquartile range) }\end{array}$ & 64 (87) & 206.5 & $0.004 *$ & $58(63)$ & $113(152)$ & 0.163 \\
\hline $\begin{array}{l}\text { White blood cell count inclusion day, } \\
\left.\times 10^{9} / 1 \text { (median interquartile range }\right)\end{array}$ & $8.5(5)$ & $11(5)$ & 0.053 & $8(5)$ & $10(5)$ & 0.096 \\
\hline $\begin{array}{l}N \text { allocated to antibiotic prophylaxis } \\
(\%)\end{array}$ & $34(50)$ & $8(36)$ & $0.193(1 \mathrm{~s})$ & $26(50)$ & $3(50)$ & $0.665(1 \mathrm{~s})$ \\
\hline
\end{tabular}

increasing resistance figures emphasises the importance of assessing the evidence basis of all indications for use of antibiotics. A recent randomised clinical trial, comparing singledose antibiotic prophylaxis with single-dose AP plus an extra dose postoperatively, in patients listed for acute cholecystectomy for mild (grade I) calculus cholecystitis, demonstrated no difference in PIC rate [21]. In three other randomised trials, continuation of prophylactic antibiotic after cholecystectomy had no significant impact on PIC rate in grades I and II cholecystitis $[6,22,23]$. The results of the present study further question the use of PAP in procedures for cholecystitis.
The use of PAP in planned cholecystectomy varies between units and regions. There is still a lack of evidence supporting the use of PAP in cases of acute cholecystectomy, but even so it is obviously a generally held view that PAP reduces PIC; otherwise, the use of PAP should not be practised to such a great extent [12].

Guidelines on antibiotic use are important for all clinicians, not only to ensure proper use of antibiotics but also to minimise its overuse. Reported proportions of ESBL-producing $E$. coli range between $31.2 \%$ in two university hospitals in Germany [24] and $70.0 \%$ in a Korean university medical

Table 3 Outcome of the cultures for those patients from whom bile samples were taken and rate PIC in relation to the samples

\begin{tabular}{|c|c|c|c|}
\hline & Positive $(n=18,37.5 \%)$ & Negative $(n=30,62.5 \%)$ & $p$ value (1 s) \\
\hline Allocation & & & 0.076 \\
\hline AP & $13(72)$ & $14(47)$ & \\
\hline Placebo & $5(28)$ & $16(53)$ & \\
\hline Postoperative infectious complication & & & 0.054 \\
\hline Non-event & $12(67)$ & $27(90)$ & \\
\hline Event & $6(33)$ & $3(10)$ & \\
\hline
\end{tabular}


centre [25]. In Europe, the rate of fully susceptible E.coli is only $41.7 \%$ and $63.4 \%$ for Klebsiella [26]. The increasing problem of drug resistance makes evidence-based guidelines even more important. In the present study, we tested piperacillin/tazobactam as prophylaxis. The use of piperacillin/tazobactam was motivated by the drug susceptibility pattern found in a previous Swedish study [11] and was one of the several drugs suggested in an update of the Tokyo guidelines on antimicrobial therapy for acute cholangitis and cholecystitis [10]. It could be argued, however, that its spectrum is too broad to be used for prophylaxis. Antibiotics with a spectrum more appropriate for prophylaxis, e.g. sulfamethoxazole/trimethoprim, could be a better alternative. Nevertheless, there are few other drugs that can be expected to be more effective as prophylaxis than piperacillin/tazobactam. Concerns other than just effectiveness must be considered when choosing an antibiotic for prophylaxis, and there is no reason to believe that the outcome of the present study would have been different if another antibiotic had been used.

As the timing of the procedure varied, the interval between study inclusions ranged from 0 to $72 \mathrm{~h}$. This may have affected the impact of AP in the PAP group, although the time from the last administration of the infusion to start of surgery never exceeded $8 \mathrm{~h}$ since the antibiotic was administrated from the time of inclusion until surgery. There was a significant correlation between CRP at baseline and PIC rate, which may have had an impact on the outcome.

It is obvious that after procedures for acute cholecystitis, the PIC rate is significant. The mechanisms behind the development of PIC are complex including factors such as bile leakage, haemorrhage and tissue damage related to surgical trauma. Intraoperative contamination with infected bile is probably the main risk factor for PIC, which is why we specifically followed contamination and bile cultures [8-10]. We found a weak association between bacterial counts in the aspirated bile and risk for PIC which was in contrast to the risk associated with conversion to an open procedure. The rate of bile culture sampling was low, which may have affected the results of this study. The higher rate of positive cultures in the placebo group is probably due to a type I error.

We defined PIC as any infectious complication requiring drainage, surgical intervention or antibiotic treatment. This definition corresponds to grade $\geq$ II complications according to the Clavien-Dindo classification, but does not include surgical site infections not requiring antibiotics. This may have led to an underestimation of the PIC rate.

The double-blinded placebo-controlled design of this study ensures high internal validity. The external validity, on the other hand, is limited by the fact that it was conducted at a single centre. The blinding procedure reduced the risk for bias in the assessment of PIC. We were obliged to prolong the inclusion period due to problems in identifying and including patients that fulfilled the criteria. Another weakness of the study is that some patients were given more than one study infusion (i.e. antibiotic or placebo) prior to surgery, due to delay of surgery.

\section{Conclusion}

PAP did not seem to affect the risk for PIC in patients with grades I and II acute cholecystitis. The major factors determining PIC in these patients must be better defined, in particular the role of bacteriobilia. The present study did not have sufficient statistical power to reveal minor reductions in PIC rate. PAP may also be motivated in subgroups with high risk for PIC. Further studies are needed to evaluate the effectiveness of PAP in patients undergoing surgery for acute cholecystitis.

Acknowledgements The skilled technical assistance of Carola Carlsson, Eugenia Furumula-Larsson, Evelina Olsson, Maura Krook and Susanne Karlsson is gratefully acknowledged.

Authors' contributions Study conception and design, FH and LL; acquisition of data, GJ and GS; analysis and interpretation of data, GJ and GS; drafting of manuscript, GJ and GS; critical revision of manuscript, all authors.

Funding Open access funding provided by Karolinska Institute. The study was supported by a grant from the Swedish Research Council (Reference 2018-06926).

Availability of data and material All data in the study are available on request from the corresponding author.

\section{Compliance with ethical standards}

Conflict of interest The authors declare that they have no conflict of interest.

Ethics approval The study protocol was approved by the Local Ethics Committee of Stockholm County (2008/1135-31) and by the Swedish Medical Product Agency (LMV EUnr 2009-010265-23 159:2009/ 41764).

Consent to participate All participants gave verbal and written consent to participation.

Open Access This article is licensed under a Creative Commons Attribution 4.0 International License, which permits use, sharing, adaptation, distribution and reproduction in any medium or format, as long as you give appropriate credit to the original author(s) and the source, provide a link to the Creative Commons licence, and indicate if changes were made. The images or other third party material in this article are included in the article's Creative Commons licence, unless indicated otherwise in a credit line to the material. If material is not included in the article's Creative Commons licence and your intended use is not permitted by statutory regulation or exceeds the permitted use, you will need to obtain permission directly from the copyright holder. To view a copy of this licence, visit http://creativecommons.org/licenses/by/4.0/. 


\section{References}

1. Lammert F, Gurusamy K, Ko CW, Miquel JF, Mendez-Sanchez N, Portincasa P et al (2016) Gallstones. Nat Rev Dis Primers 2:17

2. Khalid S, Iqbal Z, Bhatti AA (2017) Early versus delayed laparoscopic cholecystectomy for acute cholecystitis. J Ayub Med Coll Abbottabad 29(4):570-573

3. Kolla SB, Aggarwal S, Kumar A, Kumar R, Chumber S, Parshad R, Seenu V (2004) Early versus delayed laparoscopic cholecystectomy for acute cholecystitis: a prospective randomized trial. Surg Endosc 18(9):1323-1327

4. Gutt CN, Encke J, Köninger J, Harnoss JC, Weigand K, Kipfmüller K, Schunter O, Götze T, Golling MT, Menges M, Klar E, Feilhauer K, Zoller WG, Ridwelski K, Ackmann S, Baron A, Schön MR, Seitz HK, Daniel D, Stremmel W, Büchler MW (2013) Acute cholecystitis: early versus delayed cholecystectomy, a multicenter randomized trial (ACDC study, NCT00447304). Ann Surg 258(3): 385-393

5. Enochsson L, Thulin A, Osterberg J, Sandblom G, Persson G (2013) The Swedish Registry of Gallstone Surgery and Endoscopic Retrograde Cholangiopancreatography (GallRiks): a nationwide registry for quality assurance of gallstone surgery. JAMA Surg 148(5):471-478

6. Regimbeau JM, Fuks D, Pautrat K, Mauvais F, Haccart V, Msika S, Mathonnet M, Scotté M, Paquet JC, Vons C, Sielezneff I, Millat B, Chiche L, Dupont H, Duhaut P, Cossé C, Diouf M, Pocard M, FRENCH Study Group (2014) Effect of postoperative antibiotic administration on postoperative infection following cholecystectomy for acute calculous cholecystitis: a randomized clinical trial. Jama. 312(2):145-154

7. Csendes A, Burdiles P, Maluenda F, Diaz JC, Csendes P, Mitru N (1996) Simultaneous bacteriologic assessment of bile from gallbladder and common bile duct in control subjects and patients with gallstones and common duct stones. Arch Surg 131(4):389-394

8. Nitzan O, Brodsky Y, Edelstein H, Hershko D, Saliba W, Keness Y, Peretz A, Chazan B (2017) Microbiologic data in acute cholecystitis: ten years' experience from bile cultures obtained during percutaneous cholecystostomy. Surg Infect 18(3):345-349

9. Arminanzas C, Tigera T, Ferrer D, Calvo J, Herrera LA, Pajaron M et al (2016) Role of bacteriobilia in postoperative complications. Rev Esp Quimioterapia 29(3):123-129

10. Gomi H, Solomkin JS, Schlossberg D, Okamoto K, Takada T, Strasberg SM, Ukai T, Endo I, Iwashita Y, Hibi T, Pitt HA, Matsunaga N, Takamori Y, Umezawa A, Asai K, Suzuki K, Han HS, Hwang TL, Mori Y, Yoon YS, Huang WSW, Belli G, Dervenis C, Yokoe M, Kiriyama S, Itoi T, Jagannath P, Garden OJ, Miura F, de Santibañes E, Shikata S, Noguchi Y, Wada K, Honda G, Supe AN, Yoshida M, Mayumi T, Gouma DJ, Deziel DJ, Liau KH, Chen MF, Liu KH, Su CH, Chan ACW, Yoon DS, Choi IS, Jonas E, Chen XP, Fan ST, Ker CG, Giménez ME, Kitano S, Inomata M, Mukai S, Higuchi R, Hirata K, Inui K, Sumiyama Y, Yamamoto M (2018) Tokyo Guidelines 2018: antimicrobial therapy for acute cholangitis and cholecystitis. J Hepatobiliary Pancreat Sci 25(1):3-16

11. Darkahi B, Sandblom G, Liljeholm H, Videhult P, Melhus A, Rasmussen IC (2014) Biliary microflora in patients undergoing cholecystectomy. Surg Infect 15(3):262-265

12. Jaafar G, Darkahi B, Lindhagen L, Persson G, Sandblom G (2017) Disparities in the regional, hospital and individual levels of antibiotic use in gallstone surgery in Sweden. BMC Surg 17(1):128

13. Sarkut P, Kilicturgay S, Aktas H, Ozen Y, Kaya E (2017) Routine use of prophylactic antibiotics during laparoscopic cholecystectomy does not reduce the risk of surgical site infections. Surg Infect 18:603-609
14. Passos MA, Portari-Filho PE (2016) Antibiotic prophylaxis in laparoscopic cholecistectomy: is it worth doing? Arq Bras Cir Dig 29(3):170-172

15. Vohra RS, Hodson J, Pasquali S, Griffiths EA, Chole SSG (2017) West Midlands Res C. Effectiveness of antibiotic prophylaxis in non-emergency cholecystectomy using data from a populationbased cohort study. World J Surg 41(9):2231-2239

16. Murri R, De Belvis AG, Fantoni M, Tanzariello M, Parente P, Marventano $S$ et al (2016) Impact of antibiotic stewardship on perioperative antimicrobial prophylaxis. Int J Qual Health Care 28(4):502-507

17. Ansaloni L, Pisano M, Coccolini F, Peitzmann AB, Fingerhut A, Catena F, Agresta F, Allegri A, Bailey I, Balogh ZJ, Bendinelli C, Biffl W, Bonavina L, Borzellino G, Brunetti F, Burlew CC, Camapanelli G, Campanile FC, Ceresoli M, Chiara O, Civil I, Coimbra R, de Moya M, di Saverio S, Fraga GP, Gupta S, Kashuk J, Kelly MD, Khokha V, Jeekel H, Latifi R, Leppaniemi A, Maier RV, Marzi I, Moore F, Piazzalunga D, Sakakushev B, Sartelli M, Scalea T, Stahel PF, Taviloglu K, Tugnoli G, Uraneus S, Velmahos GC, Wani I, Weber DG, Viale P, Sugrue M, Ivatury R, Kluger Y, Gurusamy KS, Moore EE (2016) 2016 WSES guidelines on acute calculous cholecystitis. World J Emerg Surg 11:25

18. Liang B, Dai M, Zou ZH (2016) Safety and efficacy of antibiotic prophylaxis in patients undergoing elective laparoscopic cholecystectomy: a systematic review and meta-analysis. J Gastroenterol Hepatol 31(5):921-928

19. Bari H, Khan MR, Shariff AH (2017) Antibiotics in acute calculous cholecystitis - do Tokyo guidelines influence the surgeons' practices? J Pak Med Assoc 67(5):670-676

20. Probst P, Zaschke S, Heger P, Harnoss JC, Hüttner FJ, Mihaljevic AL, Knebel P, Diener MK (2019) Evidence-based recommendations for blinding in surgical trials. Langenbeck's Arch Surg 404(3): 273-284

21. Loozen CS, Kortram K, Kornmann VN, van Ramshorst B, Vlaminckx B, Knibbe CA et al (2017) Randomized clinical trial of extended versus single-dose perioperative antibiotic prophylaxis for acute calculous cholecystitis. Br J Surg 104(2):e151-e1e7

22. Kim EY, Yoon YC, Choi HJ, Kim KH, Park JH, Hong TH (2017) Is there a real role of postoperative antibiotic administration for mild-moderate acute cholecystitis? A prospective randomized controlled trial. J Hepatobiliary Pancreat Sci 24(10):550-558

23. de Santibanes M, Glinka J, Pelegrini P, Alvarez FA, Elizondo C, Giunta D et al (2018) Extended antibiotic therapy versus placebo after laparoscopic cholecystectomy for mild and moderate acute calculous cholecystitis: a randomized double-blind clinical trial. Surgery.

24. Reuken PA, Torres D, Baier M, Loffler B, Lubbert C, Lippmann N et al (2017) Risk Factors for multi-drug resistant pathogens and failure of empiric first-line therapy in acute cholangitis. PLoS One 12(1):e0169900

25. Kwon JS, Han J, Kim TW, Oh JH, Kwon HH, Jung JT et al (2014) Changes in causative pathogens of acute cholangitis and their antimicrobial susceptibility over a period of 6 years. The Korean journal of gastroenterology = Taehan Sohwagi Hakhoe chi 63(5):299 307

26. European Centre for Disease Prevention and Control. Antimicrobial survillance in Europe 2014. Annual Report of the European Antimicrobial Resistance Survillance Network (EARSNet) 2015

Publisher's note Springer Nature remains neutral with regard to jurisdictional claims in published maps and institutional affiliations. 